\title{
Recent results from the LHCf experiment
}

A. Tiberio ${ }^{1,2, a}$, O. Adriani ${ }^{1,2}$, E. Berti ${ }^{1,2}$, L. Bonechi ${ }^{2}$, M. Bongi ${ }^{1,2}$, G. Castellini ${ }^{2,3}$, R. D’Alessandro ${ }^{1,2}$, M. Del Prete $^{1,2}$, M. Haguenauer ${ }^{4}$, Y. Itow ${ }^{5,6}$, K. Kasahara ${ }^{7}$, K. Kawade ${ }^{5}$, Y. Makino ${ }^{5}$, K. Masuda ${ }^{5}$, E. Matsubayashi ${ }^{5}$, H. Menjo ${ }^{8}$, G. Mitsuka $^{1,5}$, Y. Muraki ${ }^{5}$, P. Papini ${ }^{2}$, A.-L. Perrot ${ }^{9}$, D. Pfeiffer ${ }^{9}$, S. Ricciarini ${ }^{2,3}$, T. Sako ${ }^{5,6}$, N. Sakurai ${ }^{6}$, Y. Shimizu ${ }^{10}$, Y.

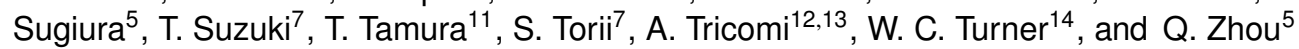

${ }^{1}$ University of Florence, Italy

${ }^{2}$ INFN Section of Florence, Italy

${ }^{3}$ IFAC-CNR, Italy

${ }^{4}$ Ecole-Polytechnique, Palaiseau, France

${ }^{5}$ Solar-Terrestrial Environment Laboratory, Nagoya University, Nagoya, Japan

${ }^{6}$ Kobayashi-Maskawa Institute for the Origin of Particles and the Universe, Nagoya University, Nagoya, Japan

${ }^{7}$ RISE, Waseda University, Japan

${ }^{8}$ Graduate school of Science, Nagoya University, Japan

${ }^{9} \mathrm{CERN}$, Switzerland

${ }^{10}$ JAXA, Japan

${ }^{11}$ Kanagawa University, Japan

${ }^{12}$ University of Catania, Italy

${ }^{13}$ INFN Section of Catania, Italy

${ }^{14}$ LBNL, Berkeley, California, USA

\begin{abstract}
The LHC-forward (LHCf) experiment, situated at the LHC accelerator, has measured neutral particles production in a very forward region (pseudo-rapidity $>8.4$ ) in proton-proton and proton-lead collisions. The main purpose of the LHCf experiment is to test hadronic interaction models used in cosmic rays experiments to simulate cosmic rays induced air-showers in Earth's atmosphere.

The experiment is composed of two independent detectors located at $140 \mathrm{~m}$ from the ATLAS interaction point (IP1) on opposite sides; each detector is composed of two sampling calorimeters.

Latest physics results from $\mathrm{p}-\mathrm{p}$ and $\mathrm{p}-\mathrm{Pb}$ collisions (at $\sqrt{s}=7 \mathrm{TeV}$ and $5.02 \mathrm{TeV}$ respectively) will be discussed in this paper; in particular, the inclusive energy spectra of neutrons in $p$-p collisions and the transverse momentum spectra of neutral pions for different pseudo-rapidity ranges in $\mathrm{p}-\mathrm{Pb}$ collisions will be shown.
\end{abstract}

\section{Introduction}

LHC-forward experiment (LHCf) has measured neutral particles production in a very forward region in protonproton and proton-lead collisions at the Large Hadron Collider. The main purpose of LHCf is to improve hadronic interaction models of Monte Carlo (MC) simulations used in cosmic rays indirect measurements. Highest energy cosmic rays can only be detected from secondary particles which are produced by the interaction of the primary particle with nuclei of the atmosphere, the so-called air showers. Studying the development of air showers, it is possible to reconstruct the type and kinematic parameters of primary particle. To reproduce the development of air showers, MC simulations with accurate hadronic interaction models are needed. Since the energy flow of secondary particles is concentrated in the forward direction, measurements of particle production at high pseudo-rapidity (i. e. small angles) are very important. Furthermore, in forward region soft QCD interactions (non perturbative regime) dominates and MC simulations of air showers are based on phenomenological model, so inputs from experimental data are crucial. LHC accelerator gives the possibility to study a wide range of collision energies, from $0.9 \mathrm{TeV}$ to $13 \mathrm{TeV}$ in the center of mass frame, which corresponds to an energy range in the laboratory frame from $10^{14} \mathrm{eV}$ to $10^{17} \mathrm{eV}$. This energy range covers the "knee" region of cosmic rays spectrum, which occurs at $10^{15} \mathrm{eV}$.

\section{The experiment}

LHCf is composed of two indipendent detectors, called Arm1 and Arm2. Arm1 is located 140 meters away from ATLAS interaction point (IP1) in the IP8 direction, while Arm2 is placed 140 meters away from IP1 in the opposite direction (toward IP2). Detectors are placed inside Target Neutral Absorber (TAN), where the beam pipe turns into two separates tubes. Since charged particles are deviated by the D1 dipole magnet (which bends colliding beams

a. e-mail: alessio.tiberio@fi.infn.it 
into the two separate beam pipes), only neutral particles, mainly photons and neutrons, reach the detector.

Each detector is made of two sampling and imaging calorimeters (called towers hereafter). Each tower is composed of 16 tungsten layers and 16 plastic scintillator layers to measure energy and also contains 4 position sensitive layers. Arm1 detector uses scintillating fiber (SciFi) to measure position, while Arm2 uses silicon microstrip detectors. Transverse cross sections of towers are $20 \times 20 \mathrm{~mm}^{2}$ and $40 \times 40 \mathrm{~mm}^{2}$ for Arm1 and $25 \times 25 \mathrm{~mm}^{2}$ and $32 \times 32 \mathrm{~mm}^{2}$ for Arm2. Longitudinal dimension of towers is of 44 radiation lengths, which correspond to 1.6 nuclear interaction lengths. Energy resolution is better than $5 \%$ for photons and of about $40 \%$ for neutrons. Position resolution for photons is $200 \mu \mathrm{m}$ and 40 $\mu \mathrm{m}$ for Arm1 and Arm2 respectively, while position resolution for neutrons is of about $1 \mathrm{~mm}$. Smaller tower of each detector is placed on the beam center and covers the pseudo-rapidity range $\eta>9.6$, while larger tower covers the pseudo-rapidity range $8.4<\eta<9.4$. More detailed descriptions of detector performance are reported elsewhere [1-3].

\section{Data analysis}

Until now, data were taken in two different runs of LHC in 2010 and 2013. In 2010 LHC produced protonproton collisions at $\sqrt{s}=900 \mathrm{GeV}$ and at $\sqrt{s}=7 \mathrm{TeV}$; in 2013 proton-lead collisions at $\sqrt{s_{N N}}=5.02 \mathrm{TeV}$ and proton-proton collisions at $\sqrt{s}=2.76 \mathrm{TeV}$ were produced. Results for single photon energy spectra and for $\pi^{0}$ transverse momentum spectra in $p-p$ collisions have already been published [4-6]. Latest analysis results are presented in this paper : preliminary results from neutron analysis in p-p collisions are shown in section 3.1 and recently published [7] results from $\pi^{0}$ analysis in $\mathrm{p}-\mathrm{Pb}$ collisions are shown in section 3.2.

\subsection{Neutrons energy spectra in p-p collisions}

In this section preliminary results from neutron analysis in p-p collisions at $\sqrt{s}=7 \mathrm{TeV}$ are presented. Data were taken during LHC Fill \# 1104 on May 15, 2010. Integrated luminosities were $0.68 \mathrm{nb}^{-1}$ and $0.53 \mathrm{nb}^{-1}$ for Arm 1 and Arm2 respectively, with a typical luminosity of $6.4 \times 10^{28} \mathrm{~cm}^{-2} \mathrm{~s}^{-1}$, derived from LHCf front counters [8].

Due to the limited dimensions of calorimeter, hadronic showers are not fully contained in the tower and the study of hadronic neutral particles is less precise and more complicated than photons. Hadronic particles (mostly neutrons) are selected using informations of longitudinal distribution of energy deposit in the calorimeter. In particular two parameters are used : the depths in the tower which contain $20 \%$ and $90 \%$ of energy deposit, called $L_{20 \%}$ and $L_{90 \%}$ respectively. A two dimensional cut on parameter $L_{2 D}=L_{90 \%}-1 / 4 \cdot L_{20 \%}$ is then applied. Impact position of particle is reconstructed by the transverse distribution of energy deposit in position sensitive layer; this is necessary to apply leakage corrections to the energy. Since the energy resolution for neutrons is of about $40 \%$, true energy spectra are smeared by detector response. Smeared energy spectra of neutrons measured by Arm1 and compared with MC simulation predictions of several models are shown in Figure 1 in three pseudo-rapidity ranges.

Unfolding technique is therefore needed to estimate true energy spectra : we use a multidimensional-spectra unfolding Bayesian method relying on energy and $P_{T}$ measured variables [9]. Preliminary results after unfolding are shown in Figure 2. It is evident from results that in the very forward pseudo-rapidity region $(\eta>10.76)$ there is a large amount of high energy neutrons, only predicted by QGSJETII-03 model. In other regions data lie between models predictions, but there isn't an unique model that represent data. High energy barions produced in forward direction lead to a small inelasticity, which implies that barions produced in the first interacion of a cosmic ray with the atmosphere carries a large fraction of primary energy : as a consequence, air shower develops deeply in the atmosphere.

\subsection{Neutral pions $P_{T}$ spectra in $\mathrm{p}-\mathrm{Pb}$ collisions}

In this section, published results for $\pi^{0}$ transverse momentum spectra in $\mathrm{p}-\mathrm{Pb}$ collisions at center of mass energy per nucleon of $\sqrt{s_{N N}}=5.02 \mathrm{TeV}$ are presented [7]. During $2013 \mathrm{p}-\mathrm{Pb}$ run, only Arm2 was installed because of two different reasons : at first, to minimize interference with ATLAS ZDC operations; then, because MC simulations predicted too high multiplicity of neutrons in $\mathrm{Pb}$-remnant side. As a consequence, Arm2 were installed on p-remnant side for almost all the time, but it also operated for some hours on the $\mathrm{Pb}$-remnant side (with the detector moved up by $4 \mathrm{~cm}$ to avoid high neutron multiplicity). Data recorded on p-remnant side in LHC Fills \# 3478 and \# 3481 on January $21-22,2013$ were used, with an integrated luminosities of $0.63 \mathrm{nb}^{-1}$.

Proton-lead collisions are very important to understand nuclear effect on forward particles production, because the first collision of a cosmic ray with the atmosphere happens between a proton and a nucleus or between two nuclei. $\pi^{0}$ events are indentified when both tower of the detector are hitten by a photon. In this analysis events with two or more particles hitting the same tower (multihit events) are rejected; however, in final spectra a correction for this cut is applied. Opening angle between photons is estimated assuming $\pi^{0}$ decay occurring at IP1 and using impact position from position sensitive layers. Knowing the energies of the two photons from calorimetric informations, it is then possible to reconstruct the invariant mass of the system. Distribution of reconstructed invariant masses in the rapidity range (in detector reference frame) $-9.4<y_{L A B}<$ -9.2 is shown in Figure 3. The peak due to $\pi^{0}$ decay is fitted with an asymmetric Gaussian, while the background is fitted with a third order polynomial. Signal events are selected when their mass fall within $3 \sigma$ from the peak (black solid lines in Figure 3). Background contribution to spectra is estimated from event which falls between 3 and $6 \sigma$ from peak (black dashed lines in Figure 3).

Unlike in $\mathrm{p}-\mathrm{p}$ collisions, in $\mathrm{p}-\mathrm{Pb}$ collisions also UltraPeripheral collisions (UPC) can occur in addition to soft 

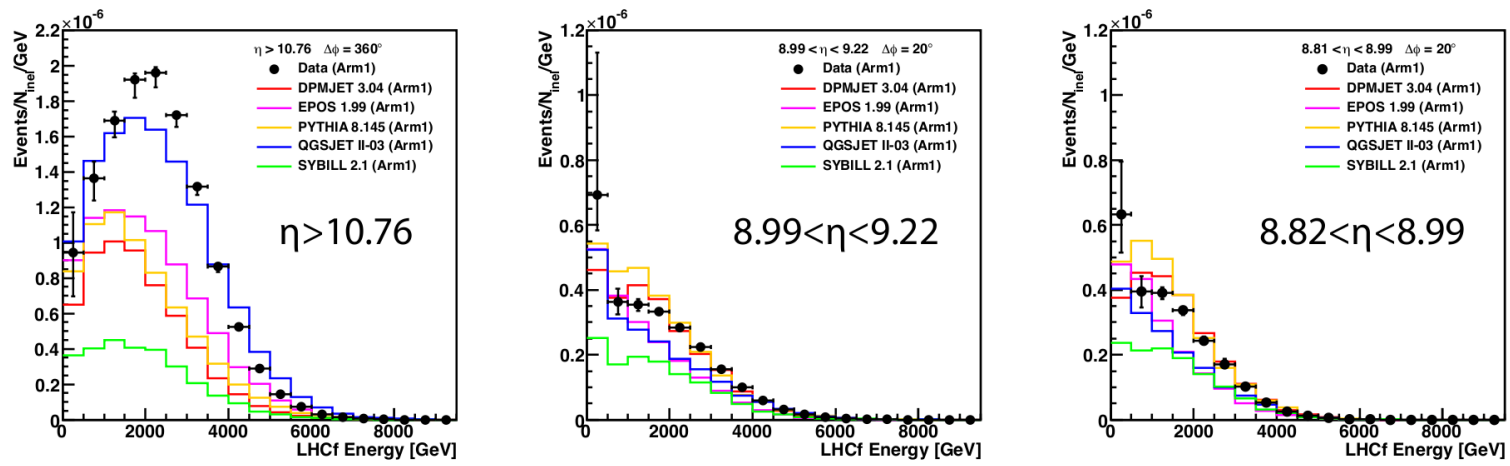

Figure 1. Energy distribution of neutrons in p-p collisions at $7 \mathrm{TeV}$ before unfolding in three pseudo-rapidity regions : coloured histograms show results from Monte Carlo simulations with different hadronic interaction models, black points show experimental data of Arm1. Particles above maximum available energy $(3500 \mathrm{GeV})$ are clearly due to the smearing effect of the detector.
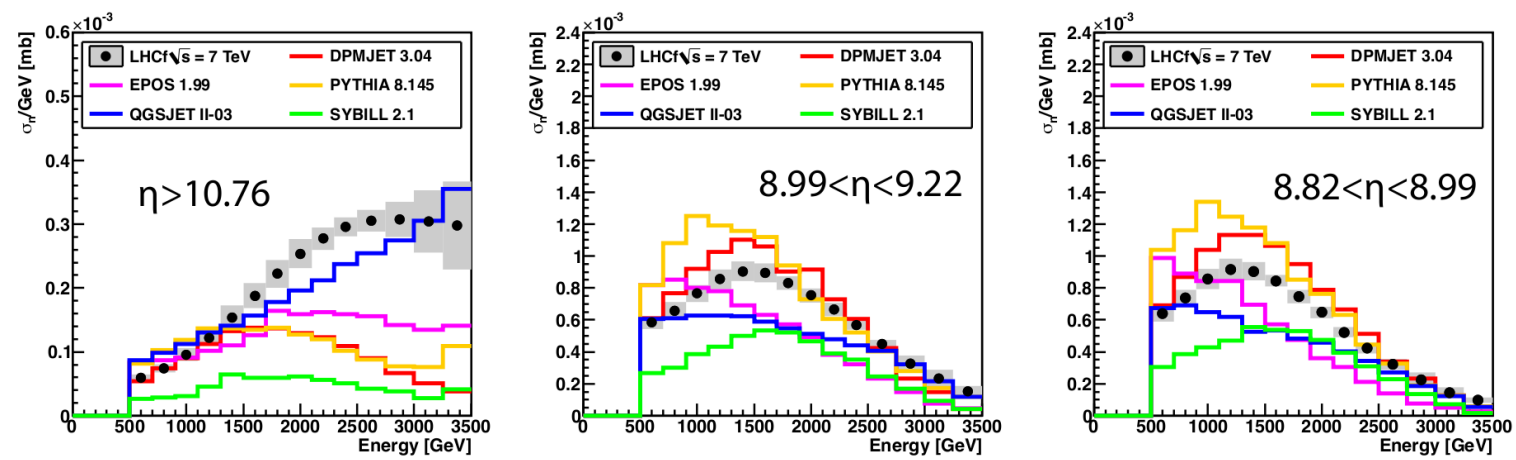

Figure 2. Energy distribution of neutrons in p-p collisions at $7 \mathrm{TeV}$ after unfolding in three pseudo-rapidity regions : coloured histograms show results from Monte Carlo simulations with different hadronic interaction models, black points show combined experimental data of Arm1 and Arm2. A large amount of forward $(\eta>10.76)$ high energy neutrons is predicted by QGSJETII-03 and confirmed by data.

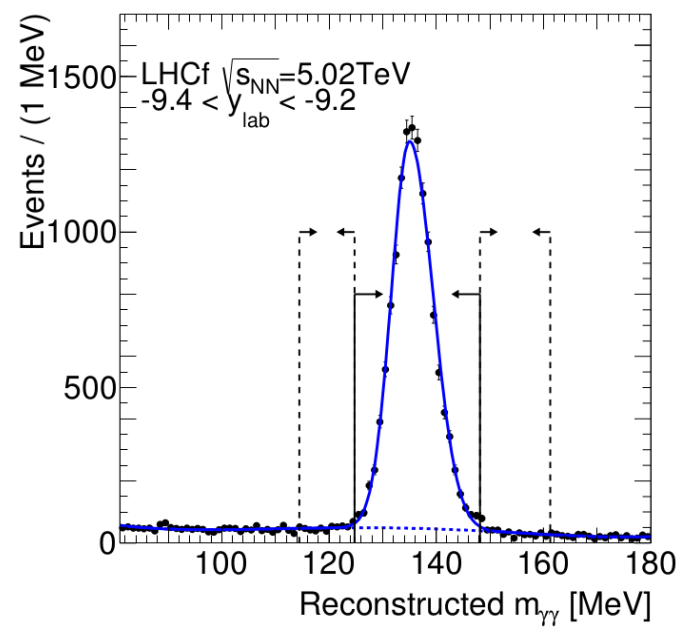

Figure 3. Two-photon invariant mass distributions in rapidity range $-9.4<y_{L A B}<-9.2$. Peak is fitted with an asymmetric Gaussian (blue solid curve), background is fitted with a polynomial (blue dashed curve). Signal and background windows are indicated by solid and dashed black lines respectively.
QCD processes. UPC occurs when a proton interacts with a virtual photon $\left(\gamma^{*}\right)$ of lead electromagnetic field [10]. UPC contribution to $\pi^{0}$ spectra is estimated from MC simulations using Weizsacker-Williams [11, 12] approximation for $\gamma^{*}$ spectrum and SOPHIA model [13] for $\mathrm{p}-\gamma^{*}$ interaction. Simulations indicate that about half of $\pi^{0}$ are generated from UPC, so this contribution must be subtracted to extract QCD informations. After subtracting UPC contributions, data are corrected for the detection efficiency and unfolded for detector response. Final spectra of data in different rapidity regions are shown and compared with MC prediction in Figure 4. Also the extrapolation at 5.02 TeV from data in p-p collisions at $0.9 \mathrm{TeV}, 2.76$ $\mathrm{TeV}$ and $7 \mathrm{TeV}$ is shown for comparison. Results indicate that spectra in $\mathrm{p}-\mathrm{Pb}$ collisions are harder than spectra in p-p collisions.

Comparing spectra in $\mathrm{p}-\mathrm{p}$ and $\mathrm{p}-\mathrm{Pb}$ collisions it is possible to estimate nuclear effects. In particular it is possible to measure the nuclear modification factor $\left(R_{p P b}\right)$ which is defined as

$$
R_{p P b} \equiv \frac{\sigma_{\text {inel }}^{p p}}{\left\langle N_{\text {coll }}\right\rangle \sigma_{\text {inel }}^{p P b}} \frac{E d^{3} \sigma^{p P b} / d p^{3}}{E d^{3} \sigma^{p p} / d p^{3}}
$$



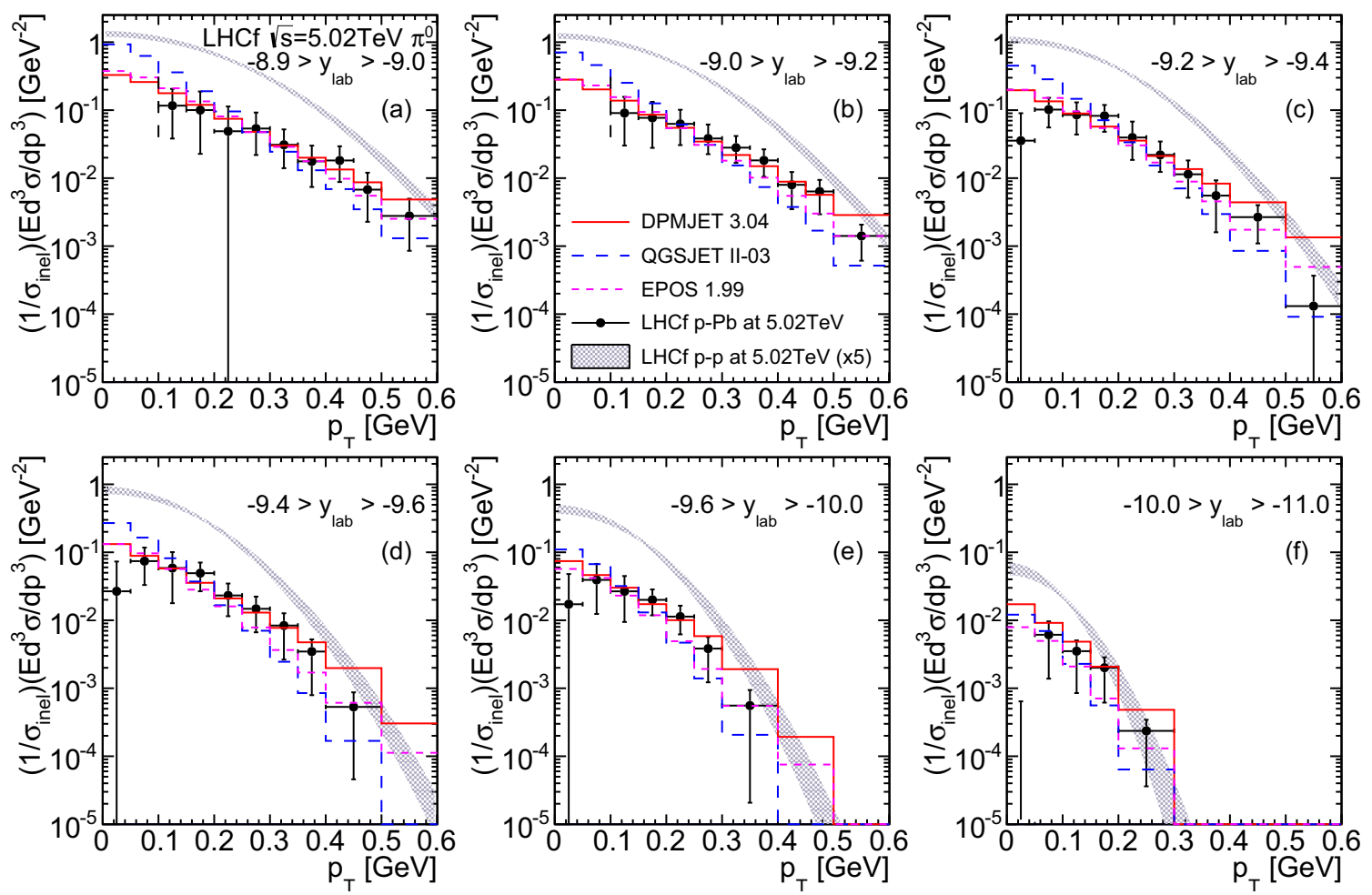

Figure 4. Transverse momentum distribution of neutral pions in several rapidity regions : coloured histograms show results from Monte Carlo simulations with different hadronic interaction models, black points show experimental data in $\mathrm{p}-\mathrm{Pb}$ collisions at $5.02 \mathrm{TeV}$, gray shaded areas represent the extrapolation at $5.02 \mathrm{TeV}$ from data with p-p collisions (multiplied by a factor 5).
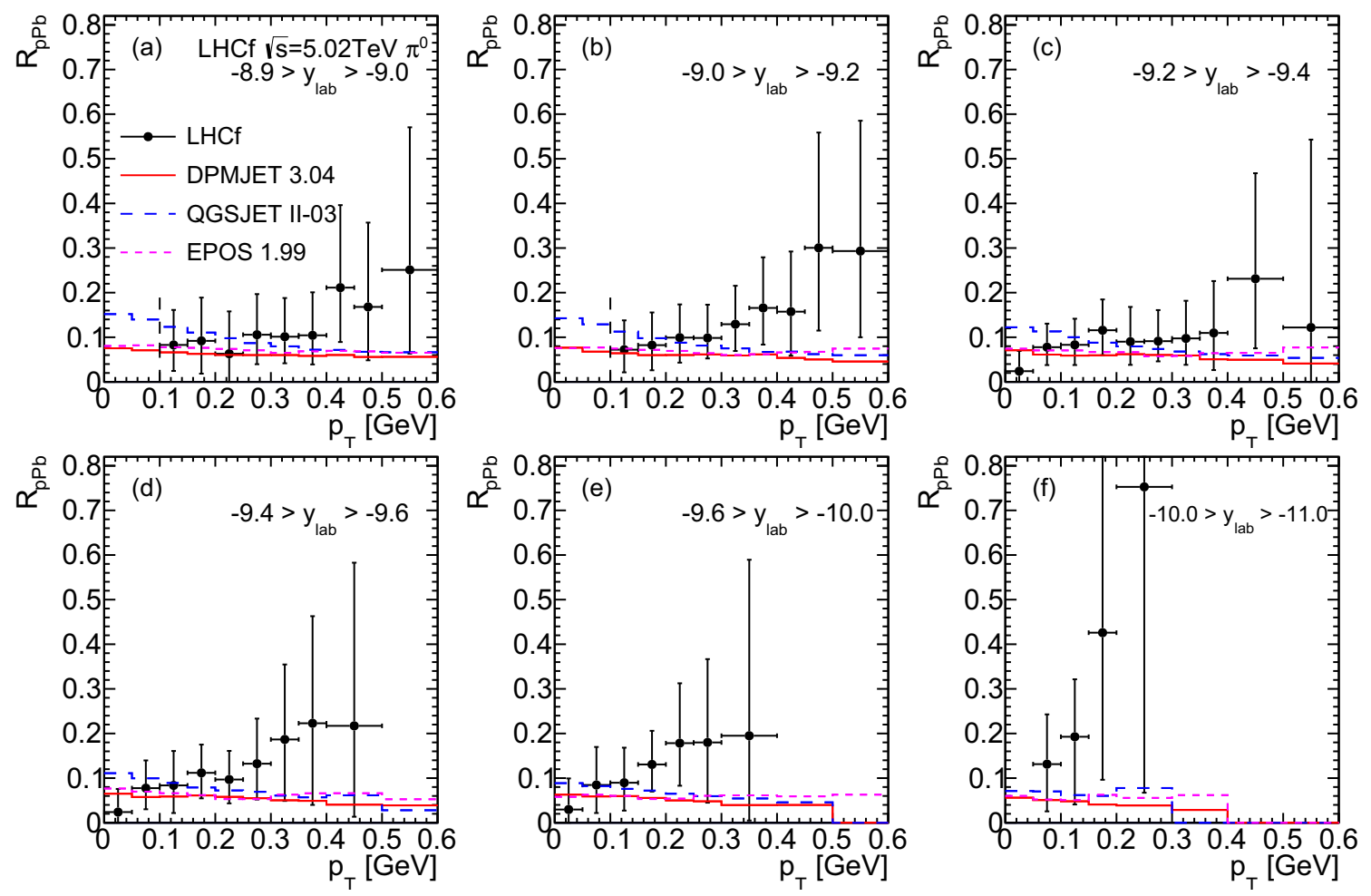

Figure 5. Nuclear modification factor for neutral pions in p-Pb collisions at $5.02 \mathrm{TeV}$ : coloured histograms show results from Monte Carlo simulations with different hadronic interaction models, experimental data are shown as black points. Nuclear modification factor grows with energy as expected. 
where $E d^{3} \sigma^{p p} / d p^{3}$ and $E d^{3} \sigma^{p P b} / d p^{3}$ are the inclusive cross sections of $\pi^{0}$ production in $\mathrm{p}-\mathrm{p}$ and $\mathrm{p}-\mathrm{Pb}$ collisions at $5.02 \mathrm{TeV}$ resspectively, and $\left\langle N_{\text {coll }}\right\rangle$ is the mean number of binary nucleon-nucleon collisions. $\left\langle N_{\text {coll }}\right\rangle$ is estimated as 6.9 from MC simulations. Nuclear modification factor as a function of $P_{T}$ in different rapidity regions is shown in Figure 5. $R_{p P b}$ grows with energy as expected, since p$\mathrm{Pb}$ spectra are harder than $\mathrm{p}-\mathrm{p}$ ones. MC models predict $R_{p P b} \sim 0.1$ over all range of $P_{T}$ and it's consistent with data within the errors.

\section{Conclusions and future prospects}

LHCf experiment performed measurements on forward production of neutral particles in proton-proton and proton-lead collisions at LHC accelerator. These measurements are necessary to calibrate hadronic interaction models used in cosmic rays physics to understand the development of atmospheric showers. LHCf performed measurements at $\sqrt{s}=0.9 \mathrm{TeV}, 2.76 \mathrm{TeV}$ and $7 \mathrm{TeV}$ in p-p collisions and at $\sqrt{s_{N N}}=5.02 \mathrm{TeV}$ in p-Pb collisions. Results from photons and $\pi^{0}$ analysis in p-p collisions are already been published. In this paper latest published results about $\pi^{0}$ analysis in $\mathrm{p}-\mathrm{Pb}$ collisions and preliminary results from neutron analysis in p-p collisions were presented. Preliminary results from neutron analysis indicate small inelasticity in the very forward region, predicted only by QGSJETII-03 model. $\pi^{0}$ analysis in $\mathrm{p}-\mathrm{Pb}$ indicates a strong suppression of $\pi^{0}$ production compared to p-p case ; nuclear modification factor from data is consistent with $\mathrm{MC}$ models predictions within errors.

Future plan for LHCf is to continue LHC operations in 2015 at $\sqrt{s}=13 \mathrm{TeV}$. Due to the higher radiation damage expected, all plastic scintillators were replaced by GSO scintillators, with more radiation hardness. Also scintillating fiber in Arm1 were replaced by GSO bars. In Arm2 silicon detectors position were changed to better catch showers in calorimeters and a new microstrip bonding scheme has been done to reduce the signal amplitude and avoid saturation of electronics with higher energy signals. In 2015 operations also common operations with ATLAS will be done. Selecting only diffractive events using ATLAS informations will be helpful to study soft QCD interactions. After 2015 operations we are proposing to bring one detector to RHIC facility; this will be useful to test Feynman scaling at lower energies and extend $P_{T}$ coverage.

\section{References}

[1] O. Adriani et al., JINST 3, S08006 (2008)

[2] O. Adriani et al., JINST 5, P01012 (2010)

[3] K. Kawade et al., JINST 9, P03016 (2014)

[4] O. Adriani et al., Physics Letters B 715, 298-303 (2012)

[5] O. Adriani et al., Physics Letters B 703, 128-134 (2011)

[6] O. Adriani et al., Physical Review D 86, 092001 (2012)

[7] O. Adriani et al., Physical Review C 89, 065209 (2014)

[8] K. Taki et al., JINST 7, T01003 (2012).

[9] G. D' Agostini, Nucl. Instrum. Meth. A 362, 487 (1995)

[10] C. A. Bertulani, S. R. Klein and J. Nystrand, Ann. Rev. of Nucl. and Part. Sci. 55, 271 (2005)

[11] C. von Weizsäcker Z. Physik 88, 612 (1934);

[12] E. J. Williams, Phys. Rev. 45, 729 (1934)

[13] A. Mücke, R. Engel, J.P. Rachen, R.J. Protheroe and T. Stanev., Comput. Phys. Comm. 124, 290 (2000) 